\title{
Photon emission in a hot QCD plasma*
}

\author{
P. Aurenche \\ January 18, 2019 \\ Laboratoire d'Annecy-le-Vieux de Physique Théorique LAPTH ${ }^{1}$, \\ BP110, F-74941, Annecy-le-Vieux Cedex, France
}

\begin{abstract}
Various mechanisms of thermal photon production are reviewed and their implications for heavy ion collisions are briefly sketched.
\end{abstract}




\section{Introduction}

It is often mentioned that photons, or more generally electromagnetic probes, can be used to signal the formation of quark-gluon plasma (QGP) in ultrarelativistic heavy ion collisions. They could also be used to measure the temperature of this plasma at the time of its formation. Data relevant for this problem will soon cover a wide energy range from a nucleon-nucleon center-of-mass energy about $20 \mathrm{GeV}$ (Pb-Pb collisions at CERN) to $200 \mathrm{Gev}$ (Au-Au collisions at RHIC, Brookhaven) and 5.5 TeV (Pb-Pb collisions at LHC after 2007).

Many production mechanisms of electromagnetic probes exist in heavy ion collisions. On a very qualitative level, one distinguishes the emission of photons in the initial stage of the collision (primary production): it is similar to the case of proton-proton collisions and goes via QCD Compton scattering, quark-antiquark annihilation and bremsstrahlung emission, involving partons in the incoming hadrons. It is calculated in perturbative QCD to the next-to-leading order (NLO). The energy spectrum is power behaved and it is expected to dominate the high momentum region. Hadrons such as $\pi^{0}$ and $\eta$ are also produced by perturbative mechanisms and they decay into photons which contribute an important background to the photon signal at large momentum. In the heavy ion collision many partons are produced which eventually thermalise into a quark-gluon plasma. This hot QGP bubble expands and cools until a hadronic phase is formed. Photons are produced in the QGP phase as well as in the hot hadronic phase with a rate which is expected to be exponentially decreasing with the energy of the photon (secondary production). This thermal contribution is expected to be important mainly in the "intermediate" energy region region up to 10 $\mathrm{GeV}$, say. Thermally produced $\pi^{0}$ 's and $\eta$ 's again contribute an important background.

In the following we review recent progress in the calculation of photon rates in the QGP phase and present phenomenological applications for RHIC and LHC heavy ion collisions.

\section{Thermal production of hard photons}

We assume in this section that the plasma is in chemical and thermal equilibrium at temperature $T$. The rate of production of a real photon of momentum $P=(E, \mathbf{p})$, per unit time and volume, is

$$
\frac{E d N}{d t d \mathbf{x} d \mathbf{p}}=-\frac{1}{(2 \pi)^{3}} n_{B}(E) \operatorname{Im} \Pi_{\mu}{ }^{\mu}(E+i \epsilon, \mathbf{p}),
$$

where $\Pi_{\mu}{ }^{\mu}(E+i \epsilon, \mathbf{p})$ is the retarded photon polarisation tensor. The BoseEinstein factor $n_{B}(E)$ provides an exponential damping when $E \gg T$. A similar formula holds for lepton pair production.

The two-point correlation function is calculated in the framework of the hard thermal loop (HTL) resummed theory of Braaten and Pisarski [1. In this approach one distinguishes two scales: the "hard" scale, typically 
of order $T$ or larger (the energy of quarks and gluons in the plasma) and the "soft" scale of order $g T$ where $g$, the strong coupling, is assumed to be small. Collective effects in the plasma modify the physics at scale $g T$, i.e. over long distances of $O(1 / g T)$. Propagators and vertices are modified by the summation of higher order diagrams. This is easily illustrated with the example of the fermion propagator, $S(Q)$, which in the "bare" theory is simply $1 / p$ (we neglect spin complications and make only a dimensional analysis $)^{2}$. The thermal contribution to the one loop correction is found to be $\Sigma(Q) \sim g^{2} T^{2} / q$ which is of $O(g T)$ when $q \sim g T$. The resummed propagator ${ }^{*} S(Q)=1 /(q-\Sigma(Q))$ is then deeply modified, compared to the bare propagator, for soft momenta of $O(g T)$ whereas thermal corrections appear essentially as higher order effects for hard momenta. Thermal resummation affects the propagator in two ways: 1$)$ in the space-like region $\left(Q^{2}<0\right)$, the propagator acquires an imaginary part, due to Landau damping (a new feature compared to the $T=0$ theory) characterised by a thermal mass scale; 2 ) in the time-like region a thermal mass, of asymptotic value $m_{\mathrm{q}}^{2}=g^{2} T^{2} C_{F} / 4$, is generated. In both cases thermal effects screen potential soft or collinear singularities. Likewise, the gluon propagator is modified: Debye screening mass $m_{\mathrm{D}}^{2}=g^{2} T^{2}\left(N_{c}+N_{f} / 2\right) / 3$ in the space-like region and quasi-particle masses in the time-like region. ${ }^{3}$ One-loop corrections also modify the vertices when the external momenta are soft [1]. One can construct an effective Lagrangian 2 2 in terms of effective propagators and vertices and calculate observables in perturbation theory.
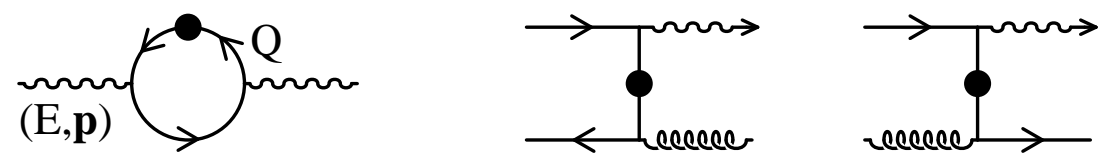

Figure 1: Left diagram: one-loop contribution; right diagrams: annihilation and Compton scattering processes obtained when cutting the one-loop diagram.

In the one-loop approximation, the production rate of hard photons is given by the diagram shown in Fig. 11 where the symbol $\bullet$ means that effective propagators are used. For hard photon momentum it is indeed enough to consider bare vertices and only one effective fermion propagator, since one propagator is necessarily hard and needs no resummation. The result is (for one quark flavor) 3

$$
\operatorname{Im} \Pi_{\mu}{ }^{\mu}(E, \mathbf{p})=\frac{5}{9} \alpha g^{2} T^{2}\left(\ln \left(\frac{E T}{m_{\mathrm{q}}^{2}}\right)+\text { constant }\right) .
$$

One notices the logaritmic growth of the 2-point function with the energy of the photon while the thermal quark mass acts as a soft cut-off which screens the singularity in the forward scattering of the processes in Fig. 1.

\footnotetext{
${ }^{2} \mathrm{~A}$ low case letter denotes the modulus of the 3-momentum.

${ }^{3} N_{c}$ is the number of colors, $N_{f}$ the number of flavors and $C_{F}$ the Casimir of the fundamental representation.
} 

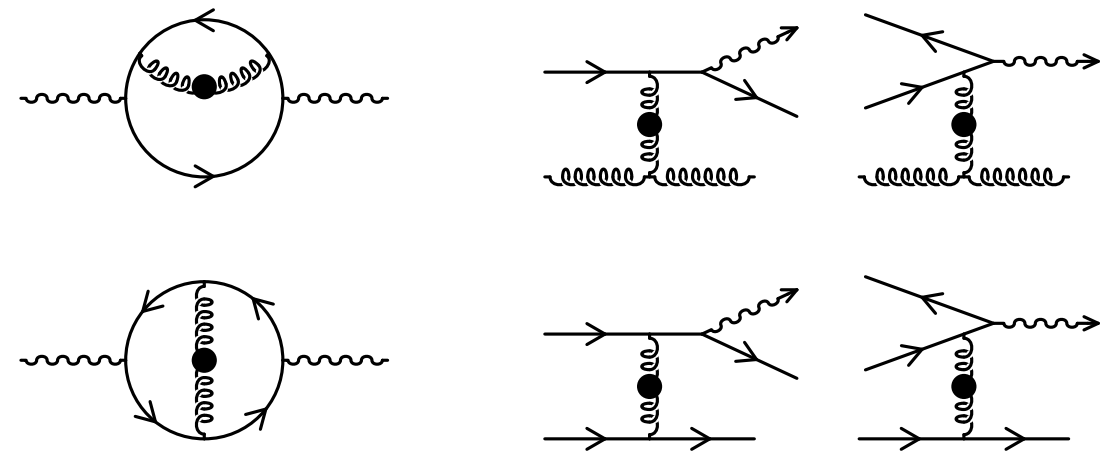

Figure 2: Left diagrams: two-loop contributions; right diagrams: bremsstrahlung and off-shell annihilation processes obtained when cutting through the two-loop diagrams.

At this point, the HTL picture of photon production, dominated by Compton and annihilation scattering, turned out to be at variance with the result of a semi-classical calculation where it was found that the production was dominated by the bremsstrahlung radiation of photons from quarks [4. The way out of this paradox is to consider the two-loop diagrams in the effective theory (Fig. 2). A dimensional analysis reveals that the dominant contribution comes from the kinematical region where the quark momenta in the loop are hard but the gluon is soft and space-like. The physical processes exhibited by cutting through the loop diagrams of Fig. 2] correspond to the bremsstrahlung emission from a quark or an anti-quark scattering in the plasma (as in the semi-classical approximation) and by a new process, the off-shell annihilation of a quark-antiquark pair where one of the (anti-)quark scatters in the plasma. For two flavors, the result of the calculation is very simple [5]:

$$
\operatorname{Im} \Pi_{\mu}{ }^{\mu}(E, \mathbf{p})=\frac{5}{9} \alpha g^{2} \frac{8}{3}\left[\frac{T^{3}}{E}+\frac{E T}{\pi^{2}}\right],
$$

where the first term arises from bremsstrahlung and the second one from offshell annihilation. In the general case the numerical coefficient is replaced by a function of the ratio of the Debye mass over the thermal quark mass [5]. The two-loop HTL result is not suppressed, as expected, by powers of the coupling $g$ compared to the one-loop result. This is due to an enhancement factor of $O\left(T^{2} / m_{\mathrm{q}}^{2}\right) \sim 1 / g^{2}$ associated to the collinear emission of the photon.

One may wonder about the usefulness of the loop expansion in the HTL effective theory if the two-loop rate is of the same order as the one-loop rate. It turns out that higher loop diagrams contribute to leading order [6]. The physical reason is the following. Consider a virtual quark of momentum $R$ decaying into a photon of momentum $P$ and a quark of momentum $Q$. The photon formation time (i.e. the life-time of the virtual state) is $\tau_{\mathrm{F}} \equiv r / R^{2} \equiv q r /\left(p\left(\mathbf{q}_{T}^{2}+m_{\mathrm{q}}^{2}\right)\right)$ with the transverse momentum of the quark measured with respect to the photon momentum. This formation time is long, of $O\left(T / m_{\mathrm{q}}^{2}\right) \sim 1 / g^{2} T$, and is comparable to the mean free path of 
the quark in the plasma given by the inverse of the quark damping rate $\gamma$. Quark rescattering in the medium should thus be taken into account. This is done by resumming the self-energy diagrams on the quark propagator going beyond the hard thermal loop approximation and including also the imaginary part (related to $\gamma$ ) generated by rescattering. In our case the time-like quark propagator, which in the HTL theory was $1 /\left(q_{T}^{2}+m_{\mathrm{q}}^{2}\right)$ becomes approximately $1 /\left(q_{T}^{2}+m_{\mathrm{q}}^{2}+i \gamma q r / p\right)$ : this shows that both thermal mass and damping rate act as competing cut-offs. Of course, to respect

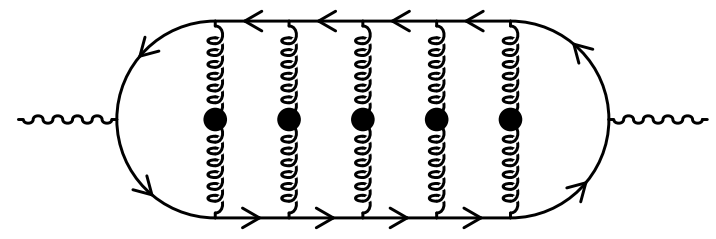

Figure 3: An example of a ladder diagram.

gauge invariance, one should resum vertex corrections in the same approximation as self-energy corrections [7, which motivates the fact that ladder diagrams such as the graph of Fig. 3 should be summed. Arnold, Moore and Yaffe [7] showed that such diagrams indeed contribute to leading order and they derived a very elegant integral equation to perform the resummation. The imaginary part of the photon polarisation tensor takes the form:

$\operatorname{Im} \Pi_{\mu}{ }^{\mu}(P) \approx \alpha N_{c} \int_{-\infty}^{+\infty} d q\left[n_{F}(r)-n_{F}(q)\right] \frac{q^{2}+r^{2}}{q^{2} r^{2}} \operatorname{Re} \int \frac{d^{2} \mathbf{q}_{T}}{(2 \pi)^{2}} \mathbf{q}_{T} \cdot \mathbf{f}\left(\mathbf{q}_{T}\right)$,

with $r=p+q$ and $n_{F}(q)=1 /(\exp (q / T)+1)$, the Fermi-Dirac statistical weight. The dimensionless resummed quark-quark-photon vertex, $\mathbf{f}\left(\mathbf{q}_{T}\right)$, is shown by the shaded vertex in Fig. 4 while $\mathbf{q}_{T}$ is proportional to the bare vertex. The value of $\mathbf{f}\left(\mathbf{q}_{T}\right)$ is obtained by solving the following equation [7]:
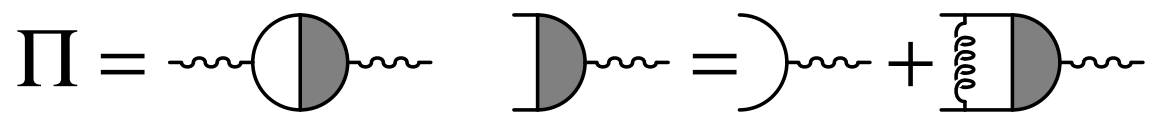

Figure 4: The integral equation resumming the ladder diagrams.

$$
\frac{i}{2 \tau_{\mathrm{F}}} \mathbf{f}\left(\mathbf{q}_{T}\right)=2 \mathbf{q}_{T}+g^{2} C_{F} T \int \frac{d^{2} \mathbf{l}_{T}}{(2 \pi)^{2}} C\left(\mathbf{l}_{T}\right)\left[\mathbf{f}\left(\mathbf{q}_{T}+\mathbf{l}_{T}\right)-\mathbf{f}\left(\mathbf{q}_{T}\right)\right],
$$

where $\tau_{\mathrm{F}}$ is the formation time defined above. The collision kernel is $C\left(\mathbf{l}_{T}\right)=$ $m_{\mathrm{D}}^{2} / \mathbf{l}_{T}^{2}\left(\mathbf{l}_{T}^{2}+m_{\mathrm{D}}^{2}\right)$ and it includes the exchange of both longitudinal and transverse gluons [6]. Solving for this integral equation one can obtain a fit, in the large $E / T$ range

$$
\operatorname{Im} \Pi_{\mu}^{\mu}(P) \approx \alpha g^{2} T^{2} \sqrt{\frac{E}{T}}
$$




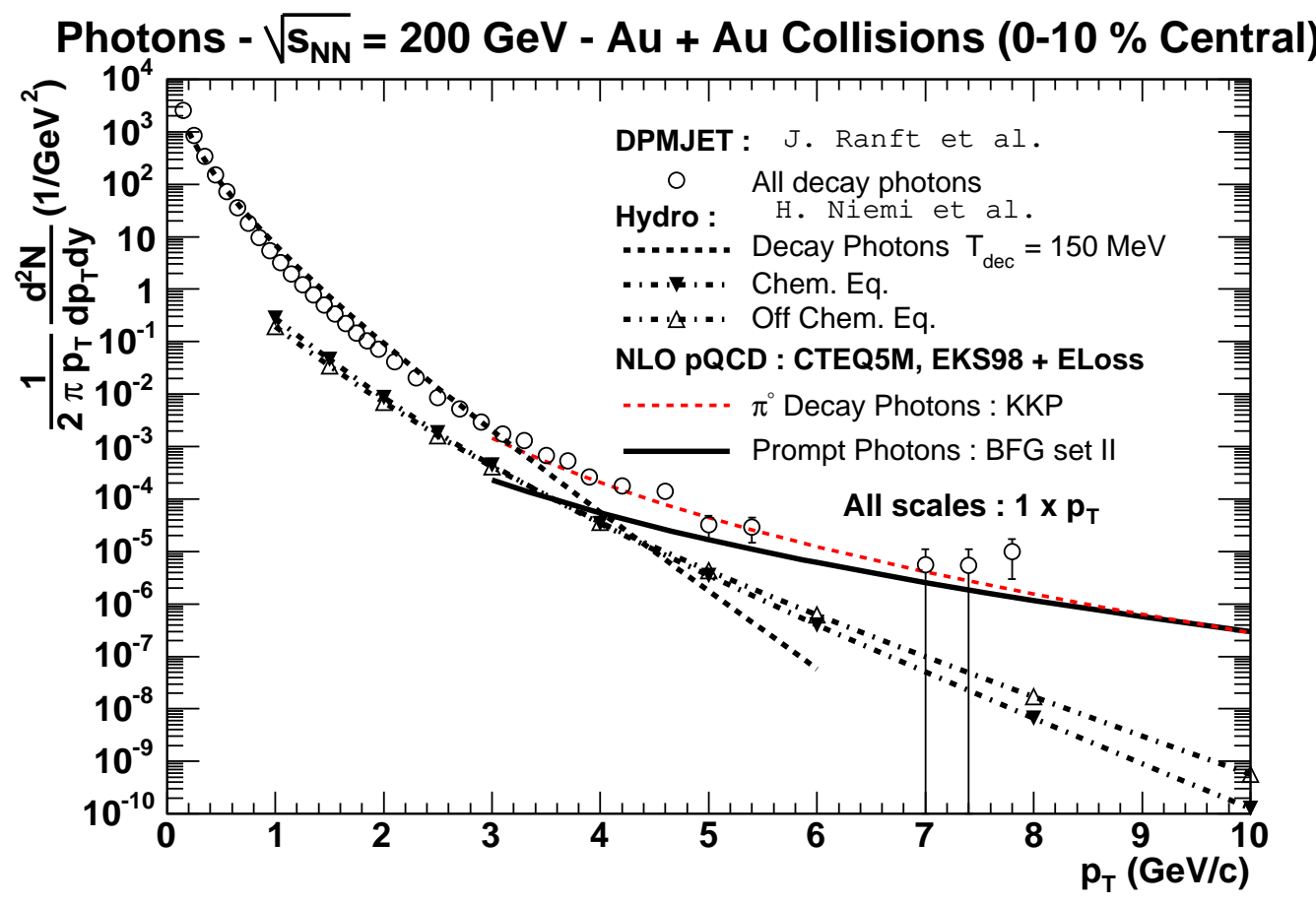

Figure 5: Various contributions to the production of photons in $\mathrm{Au}-\mathrm{Au}$ collisions at RHIC. The open dots are the predictions of decay photons from the non-thermal DPMJET model [12] for heavy ion collisions, in good agreement with the hydrodynamic + perturbative QCD model.

to be compared to the linear in $E$ behaviour of Eq. (3). This is an illustration of the Landau-Pomeranchuck-Migdal (LPM) suppression of hard photons due to multiple scattering of the quark in the plasma.

Rates for small mass lepton pairs produced at large momentum have also been calculated at one-loop [8, two-loop 9] and including the LPM effect [10.

\section{Phenomenology at RHIC and LHC}

To make predictions for photon production in heavy ion collisions, the rates calculated above have to be included in a hydrodynamical code which describes the expansion and the cooling of the plasma from chosen initial state conditions. In the following we use the code of Ruuskanen et al. 11. The rate for thermal photon production is shown by the thick dash-dotted line in Fig. 5 for Au-Au central collisions at RHIC and in Fig. 6 for $\mathrm{Pb}-\mathrm{Pb}$ central collisons at LHC. Further details on the model can be found in Ref. 13. Also shown on the figures are the rates of production of decay photons from thermally produced resonances (mainly $\pi^{0}$ and $\eta$ ) (thick dashed lines) which dominate in the low $p_{T}$ range. We also display the rates of primary photons produced in perturbative QCD mechanisms (solid lines) contributing in the large $p_{T}$ range as well as the decay photons from perturbatively produced 
resonances. In these estimates account has been taken of energy loss effects on the jet fragmentation in the medium using the model of Ref. [14.

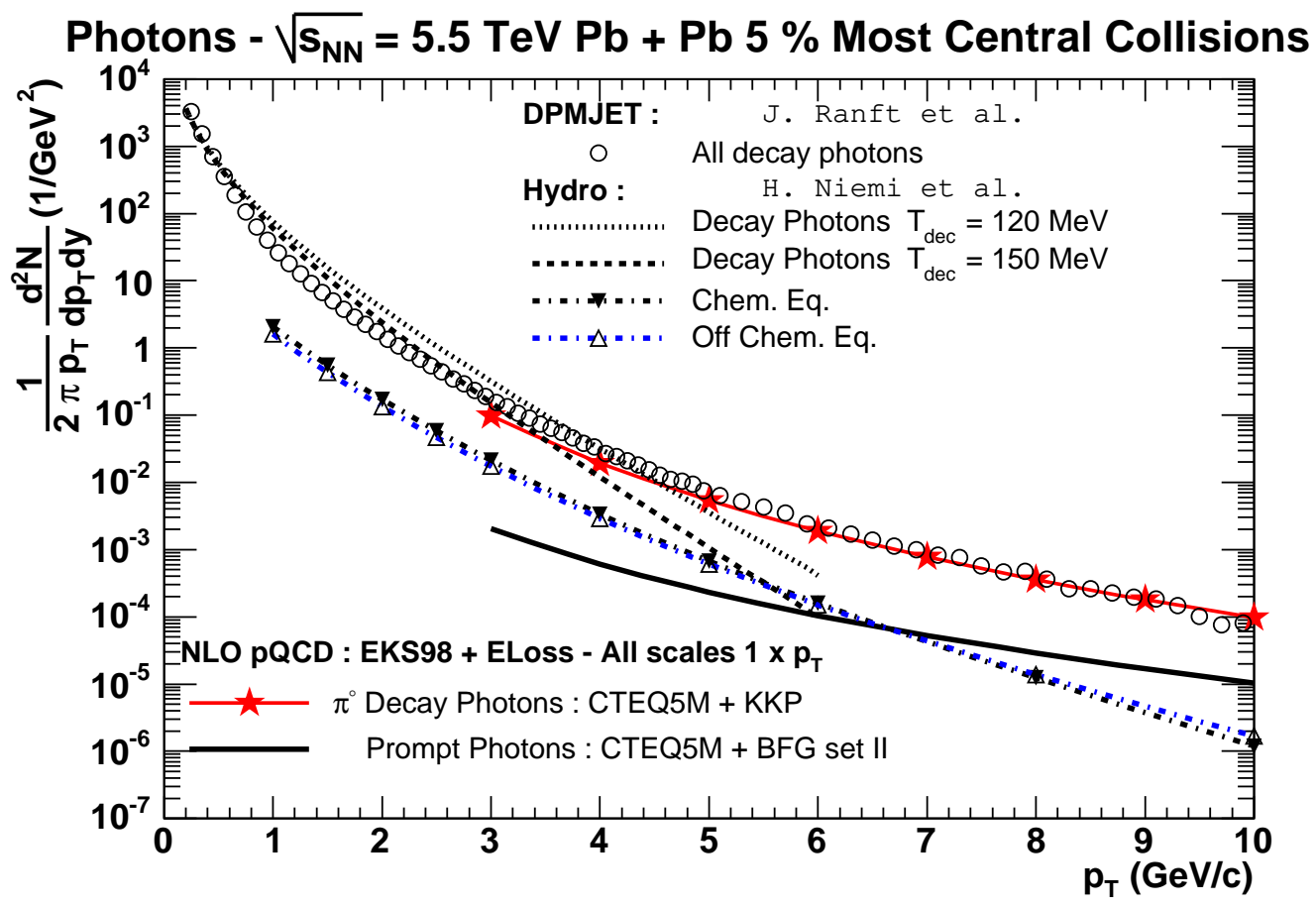

Figure 6: Same as the above figure for $\mathrm{Pb}-\mathrm{Pb}$ collisions at LHC.

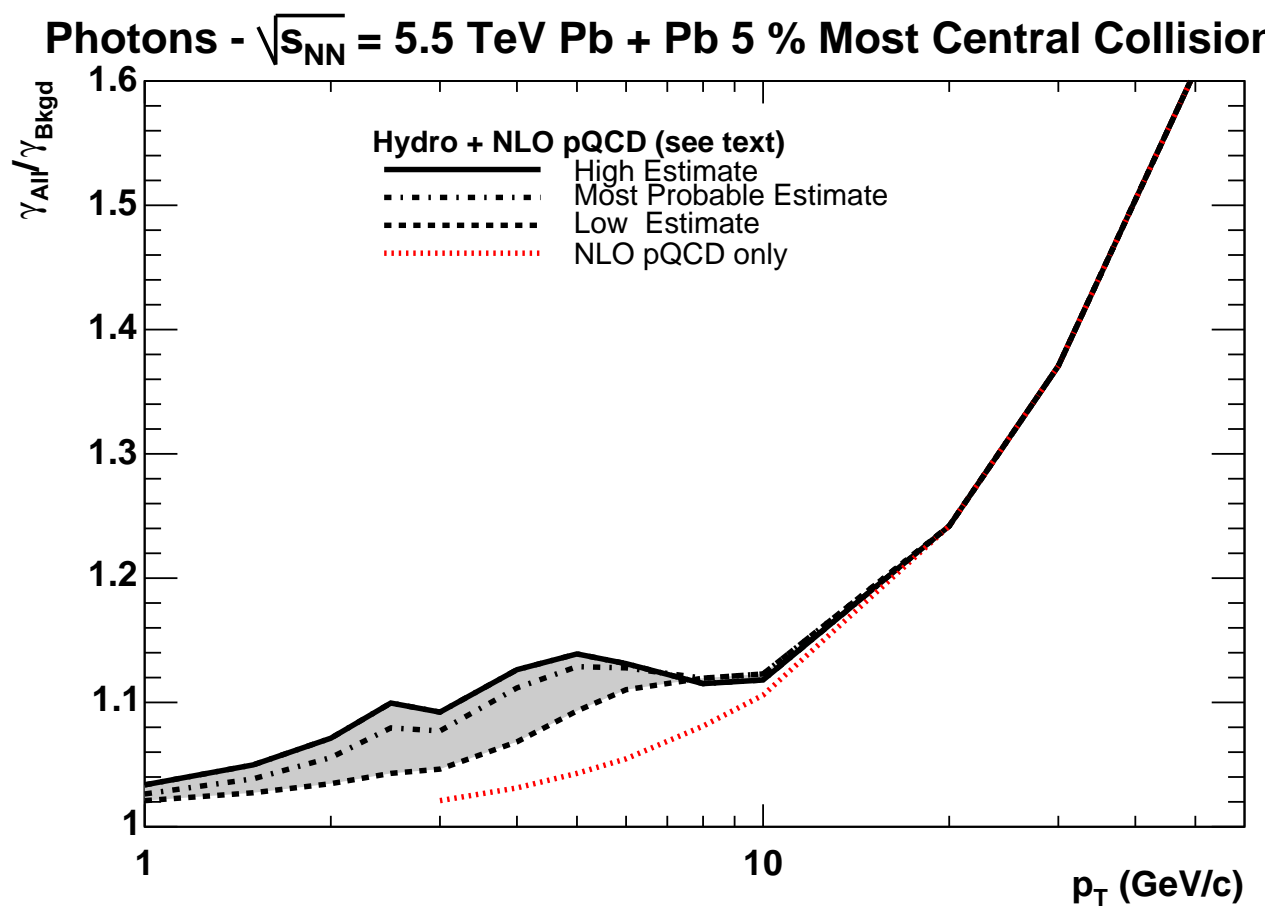

Figure 7: Ratio of all photons over decay photons at LHC with thermal production (thick lines) and without thermal production. 
It is instructive to look at the ratio of "all" produced photons over decay photons and to compare it with the prediction for the same ratio assuming no thermal production. This plot quantifies the excess of direct photons produced by the hot hadronic medium formed in a heavy ion collision. At LHC, thermal effects are important but the ratio of directly produced photon remains only about $10 \%$.

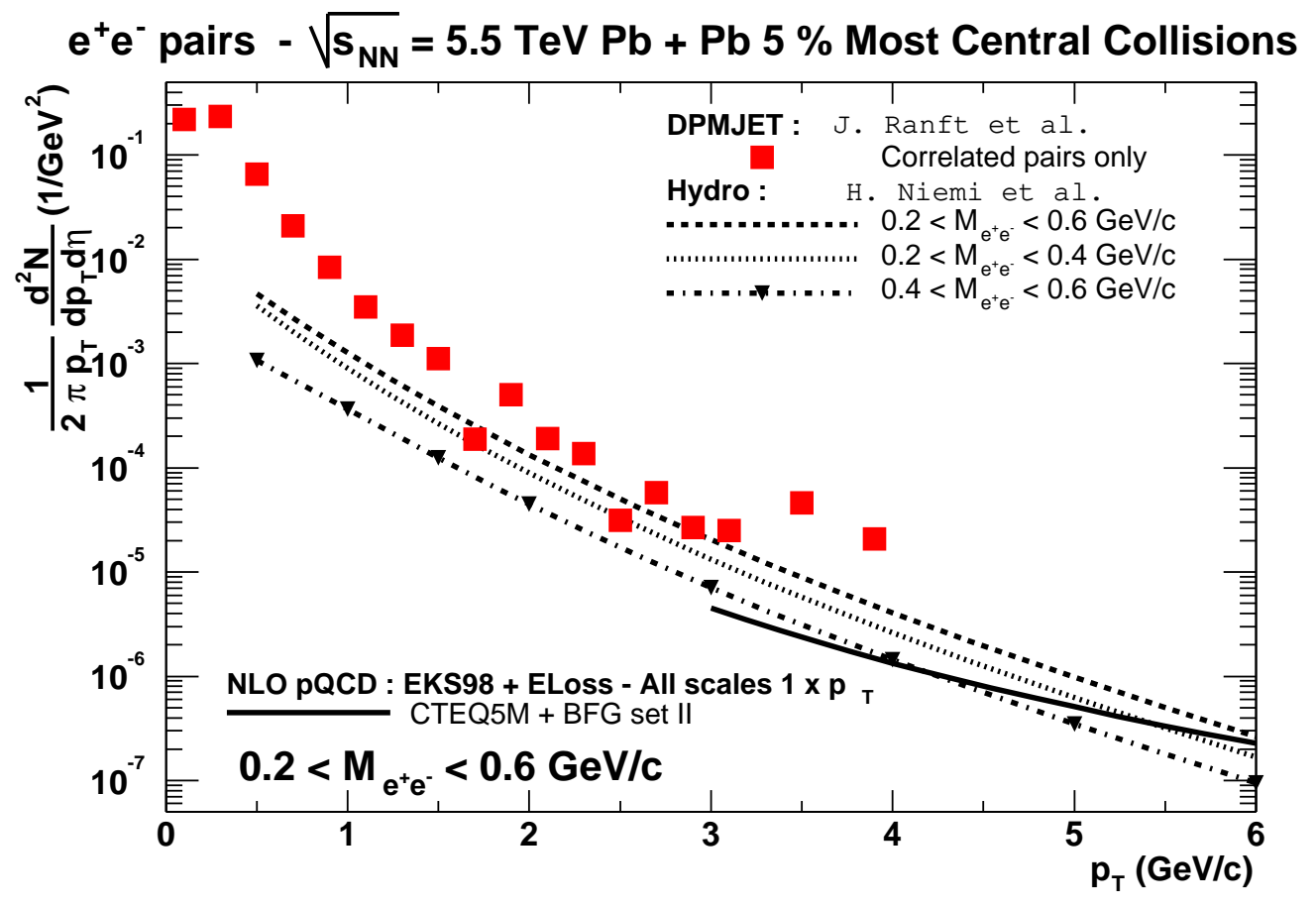

Figure 8: Thermally produced lepton pairs (dashed line) compared to perturbatively produced pairs (solid line) and background from hadronic decays (squares).

A channel which should suffer less background than direct photon is that of small mass lepton pair at large momentum: indeed choosing a pair mass above the $\pi^{0}$ mass should eliminate a large background. The thermal production rates have been calculated and included in the hydrodynamical code [13. The result is shown in Fig. 8.

\section{Further developments}

In the discussion above we have considered two independent production processes: emission of the photon in the initial stages of the collision and production in the quark-gluon plasma and hot hadronic phase. One can envisage "mixed" production mechanisms where a photon is emitted in the interaction of a hard quark or gluon (calculable in perturbative QCD) with a quark or the gluon of the plasma. Since the rate of hard partons is power behaved while thermal interactions are exponentially damped, the resulting rate of photon production is expected to be power behaved and to domi- 
nate over purely thermal processes at large momentum. Such examples of "mixed" interactions have been considered in Ref. 15] who made simplified estimates of the Compton $(g q \rightarrow \gamma q)$ and annihilation $(q \bar{q} \rightarrow \gamma g)$ processes when one of the initial $q$ or $g$ is hard and the other parton is thermalised. The result is shown in Fig. 9 by the dashed line to be compared with the dot-

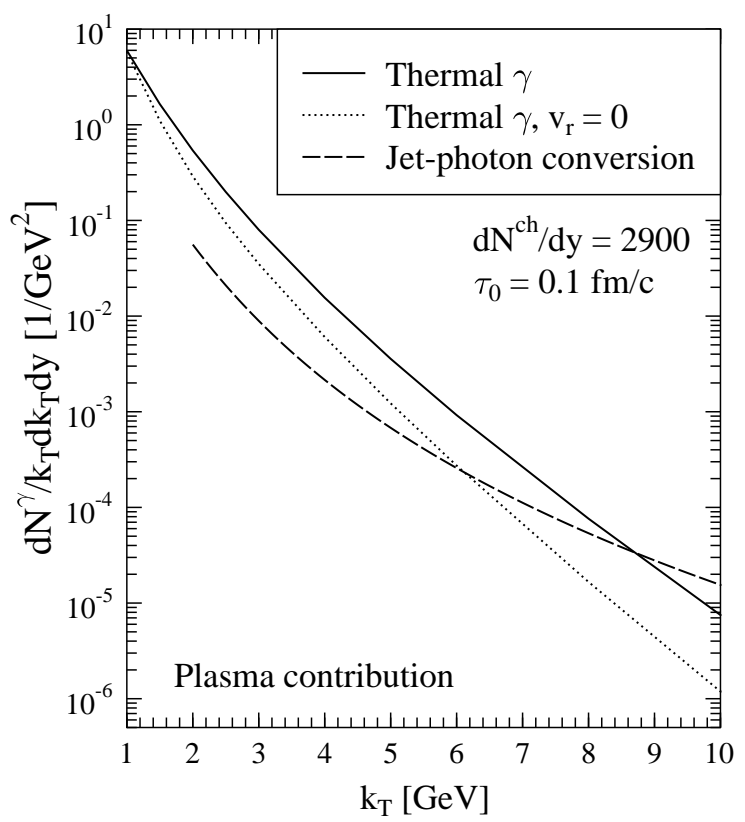

Figure 9: Photon production from "mixed" processes: rate from the Compton and annihilation processes between a hard parton with a parton in the plasma (dashed line); thermal photon production under the same hypothesis of no transverse expansion (dotted line). For comparison the thermal rate with radial expansion, used in the previous section, is also shown (solid line).

ted line which is the estimate of the purely thermal process under the same hydrodynamical conditions (no radial expansion). Recently B. Zakharov estimated the enhanced bremsstrahlung emission of a photon by a hard quark traversing the plasma [16]. He found that, due to finite size effects, the usual $1 / x$ spectrum characteristic of bremsstrahlung of a quark in vacuum is replaced by an approximate $1 /(1-x)$ spectrum for $x<1$. This is illustrated in Fig. 10 which compares the fragmentation function of a quark into a photon in vacuum with the same in the medium taking into account only one rescattering in the plasma. The change of shape of the spectrum will lead to an enhanced production of photon by bremsstrahlung compared to production in the vacuum. Multiple scattering effects will somewhat reduce the size of the predicted enhancement [16]. In the figures of the previous section we already included medium effects on bremsstrahlung production of photons and this lead to a suppression of photon emission compared to vacuum which seems to contradict the present result. In fact, there is no contradiction if we consider that in the latter case the photon was emitted by the quark outside the plasma while the former case one estimates the emission of the photon in the plasma.

It would be important to have a complete and consistent estimate of the mixed production rates, using a realistic hydrodynamical model: in any case, they will increase the thermal production rates calculated in the previous 

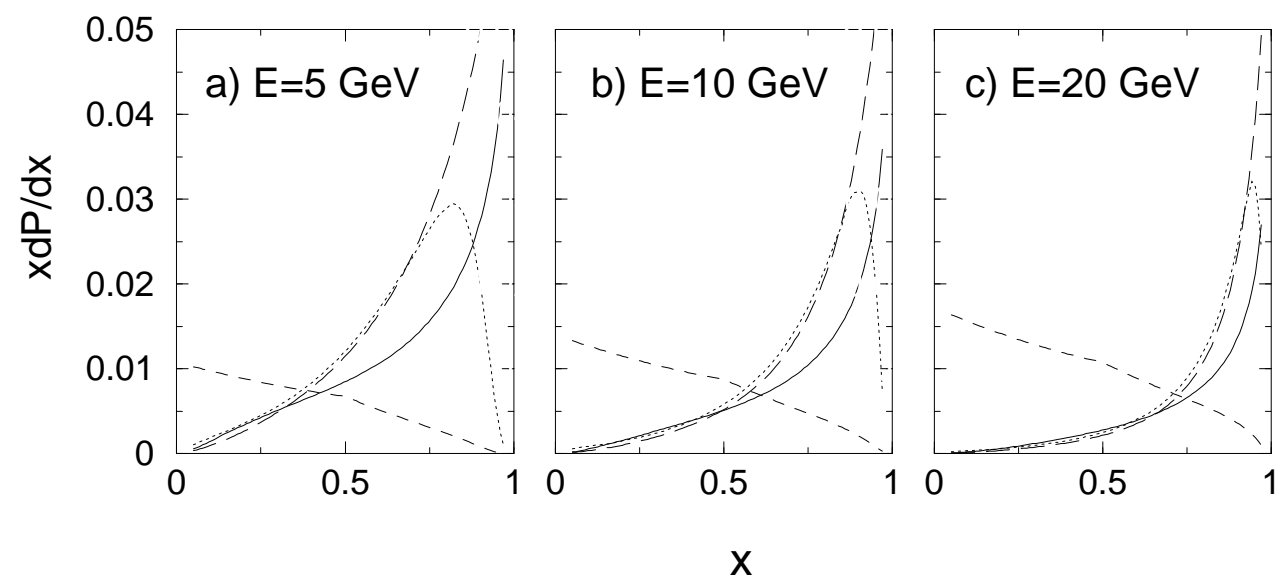

Figure 10: Comparison of the fragmentation function of a quark into a photon in vacuum (short dashed line) and in the medium (dotted line) with one scattering of the hard quark in the plasma. The other curves are for an infinite medium.

section.

All previous results are obtained assuming local thermal equilibrium and the rates are calculated per unit time. In a series of papers Boyanovsky et al [17] proposed a model to explicitely account for the finite life-time of the plasma. They assume that photon emission starts at an initial time $t_{i}$ and ends at time $t_{f}$ : under this assumption, usually forbidden processes of type $q \rightarrow q \gamma$ contribute since energy conservation does not hold. As a consequence a transient term of type $\alpha \ln t$, where $t=t_{f}-t_{i}$, appears besides the usual $\alpha \alpha_{s} t$ term, and it is claimed to dominate the photon production rate at LHC at large enough momenta. Several objections have been raised concerning the validity of this picture [18, 19, 20. In particular the hypothesis of a sharp switching on and off of the interactions is probably not adequate for the description of the physics of the problem.

\section{Acknowledgments}

I thank F. Gelis and V. Ruuskanen for collaboration and many discussions. I also thank F. Gelis for a careful reading of the manuscript.

\section{References}

[1] E. Braaten, R.D. Pisarski, Nucl. Phys. B337, 569 (1990); B339, 310 (1990); J. Frenkel, J.C. Taylor, Nucl. Phys. B334, 199 (1990); B374, 156 (1992).

[2] J.C. Taylor, S.M.H. Wong, Nuc. Phys. B346, 115 (1990); E. Braaten, R.D. Pisarski, Phys. Rev. D45, 1827 (1992). 
[3] J.I. Kapusta, P. Lichard, D. Seibert, Phys. Rev. D44 (1991) 2774; R. Baier, H. Nakkagawa, A. Niegawa, K. Redlich, Z. Phys. C53, (1992) 433.

[4] J. Cleymans, V.V. Goloviznin, K. Redlich, Phys. Rev. D47, 989 (1993); V.V. Goloviznin, K. Redlich, Phys. Lett. B 319, 520 (1993).

[5] P. Aurenche, F. Gelis, R. Kobes, H. Zaraket, Phys. Rev. D58, 085003 (1998); P. Aurenche, F. Gelis, H. Zaraket, JHEP 0205, 043 (2002).

[6] P. Aurenche, F. Gelis, H. Zaraket, Phys. Rev. D61, 116001 (2000); D62, 096012 (2000).

[7] P. Arnold, G.D. Moore, L.G. Yaffe, JHEP 0111, 057 (2001); JHEP 0112, 009 (2001); JHEP 0206, 030 (2002).

[8] T. Altherr, P.V. Ruuskanen, Nucl. Phys. B380, 377 (1992); M.H. Thoma, C.T. Traxler, Phys. Rev. D56, 198 (1997).

[9] P. Aurenche, F. Gelis, H. Zaraket, JHEP 0207, 063 (2002).

[10] P. Aurenche, F. Gelis, G.D. Moore, H. Zaraket, JHEP 0212, 006 (2002).

[11] K.J. Eskola, K. Kajantie, P.V. Ruuskanen, Eur. Phys.J. C1, 627 (1998); K.J. Eskola, P.V. Ruuskanen, S.S. Räsänen, K. Tuominen, Nucl. Phys. A696, 715 (2001); K.J. Eskola, H. Niemi, P.V. Ruuskanen, S.S. Räsänen, Nucl. Phys. A715, 561 (2003); K.J. Eskola, H. Niemi, P.V. Ruuskanen, Phys. Lett. B566, 187 (2003).

[12] R. Engel and J. Ranft, Phys. Rev. D54, 4244 (1996); S. Roesler, R. Engel and J. Ranft, hep-ph/0012252; J. Ranft, hep-ph/9911213, hep-ph/9911232.

[13] F. Arleo et al., Photon physics in heavy ion collisions at the LHC, hep-ph/0311131, to appear in the CERN Yellow Report on Hard probes in heavy ion collisions at the LHC.

[14] J. Jalilian-Marian, K. Orginos and I. Sarcevic, Phys. Rev. C63, 041901 (2001); ibid. A700, 523 (2002); S. Jeon, J. Jalilian-Marian and I. Sarcevic, Phys. Lett. B562, 45 (2003).

[15] R. J. Fries, B. Muller and D. K. Srivastava, Phys. Rev. Lett. 90, 132301 (2003).

[16] B.G. Zakharov, hep-ph/0405101.

[17] D. Boyanovsky, H. J. de Vega, Phys. Rev. D68, 065018 (2003); hep-ph/0311156.

[18] I. Dadic, F. Gelis, G.D. Moore, J. Serreau in Ref. [13], Sec. 9.

[19] J. Serreau, JHEP 0405, 078 (2004).

[20] E. Fraga, F. Gelis, D. Schiff, hep-ph/0312222 